\title{
Estimation of Different Genetic Parameters in Various Safflower (Carthamus tinctorius L.) Genotypes under Field Condition
}

\author{
Rao Wali Muhammad ${ }^{1}$, Hafiz Muhammad Wasif Ali ${ }^{2 *}$, Amir Hamza ${ }^{1}$, Muhammad Qadir Ahmad $^{2}$, Abdul \\ Qayyum $^{2}$, Waqas Malik ${ }^{2}$ and Etrat Noor ${ }^{2}$
}

${ }^{1}$ PARC Research and Training Station, Pakistan Agriculture Research Council, Multan, Pakistan; ${ }^{2}$ Department of Plant Breeding and Genetics, FAST, Bahauddin Zakariya University, Multan, Pakistan; ${ }^{3}$ Arid Zone Research Institute, Pakistan Agriculture Research Council, Bahawalpur, Pakistan.

Abstract | The study was carried out to investigate the genetic estimates regarding various economic traits i.e. days to flowering, plant height, days to maturity, no. of primary branches per plant, no. of secondary branches per plant, pods per plant, 1000-seed weight and yield per plant of 200 safflower accessions. Analysis of variance and principal component analysis were carried out to estimate the extent of variability for studied parameters and to partition the germplasm into various cluster groups on basis of their mean performance. A value of $74 \%$ was indicated by the first three PCs, which showed the highest variability between studied parameters. Eigen values $>1$ contributed variability between the genotypes under field condition. Minimum and maximum heritability estimates ranged between $77.4 \%-99.0 \%$ for days to flowering and pods per plant, respectively. Genetic advance estimates ranged between $11.99-27.21 \%$ for number of primary branches per plant and yield per plant, respectively. However, coefficient of variance ranged between $2.67-19.31 \%$ for days to maturity and number of primary branches per plant. High broad sense heritability magnitudes predicted that studied parameters were under influence of additive genetic effects and less affected by environment. Thus, direct selection of accessions on basis of studied parameters could lead to genetic improvement of the material and these traits could also be helpful for potential improvement of yield in safflower (Carthamus tinctorius).

Received | February 17, 2020; Accepted | September 18, 2020; Published | October 17, 2020

*Correspondence | Hafiz Muhammad Wasif Ali, Department of Plant Breeding and Genetics, FAST, Bahauddin Zakariya University, Multan, Pakistan; Email: wasifdogar@gmail.com

Citation | Muhammad, R.W., H.M.W. Ali, A. Hamza, M.Q.Ahmad, A. Qayyum, W. Malik and E. Noor. 2020. Estimation of different genetic parameters in various safflower (Carthamus tinctorius L.) genotypes under field condition. Pakistan Journal of Agricultural Research, 33 (4): 849-857. DOI | http://dx.doi.org/10.17582/journal.pjar/2020/33.4.849.857

Keywords | Safflower, Genetic correlation, Heritability, Phenotypic correlation, PCA

\section{Introduction}

Safflower is famous oil seed crop of ancient world

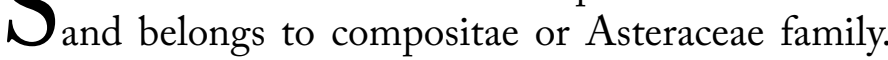
It is a good source of high-quality oil mostly used for industrial, ornamental, biofuel and food purposes (Sehgal et al., 2009; Canavar et al., 2014). It is a multipurpose crop, mostly cultivated as cut flower, medicinal plant, vegetable crop, fodder crop, dye and oil extracting source for paint industry (Emongor,
2010; Emongor et al., 2015).

Safflower oil is rich source of vitamin ' $E$ ', polyunsaturated (linoleic acid) and monounsaturated (oleic acid) fatty acids, which are helpful in lowering blood cholesterol level (Baydar and Turgot, 1999; Arslan et al., 2003). Percent concentration of linoleum acid (70-87\%) and oleic acid (11-87\%) is much high as compared to olive oil, peanut, soybean, cotton seed and corn oil (Reza et al., 2013). Oil is also used in 
preparation of soft margarines and salad oil (Conge et al., 2007). Safflower seeds are rich in vitamins, minerals and tocopherols (Velasco et al., 2005). Petals of flower are used in manufacturing of dyes, food color and medicines (Istanbulluoglu, 2009; Emongor, 2010).

The crop was mainly cultivated in arid and semiarid regions of the world with low irrigation, low fertilizer input and on marginal lands (Hojati et al., 2011). In past, safflower was cultivated on limited area, as minor crop (Canavar et al., 2014). Now, the scenario has changed. Efforts have been made to raise the cultivated area and productivity of the crop all over the world. Naturally, safflower is a temperate zone crop, but has capability of bearing temperature ranges from -7 to $40{ }^{\circ} \mathrm{C}$ with zero frost injury during vegetative and flowering growth periods. Crop is widely grown in more than 60 countries of the world, being resistance to many abiotic stresses. India, China, USA, Ethiopia, Kenya, Mexico, Argentina, Australia, Canada, Italy, Spain, Turkey, Iraq, Syria, Kazakhstan, Iran, Uzbekistan, Morocco, Israel, Russia and Pakistan are the commercial growers of the Safflower in the world (Emongor and Oagile, 2017).

Cropping system of Pakistan is deficit in space for cultivation of both conventional and non-conventional oil seed crops and these are considered as minor crops. However, to meet the requirement of vegetable oil for humans, animals and industry, rearing of conventional oil seed crops over limited area is not fruitful. So, it is a dire need of the time to motivate the growers for sowing of non-conventional oil seed crops in Pakistan. Northern areas of Sindh and Baluchistan are suitable for cultivation of safflower as an oil seed crop. While, in Punjab and KPK provinces, arid and semi-arid regions have favorable environmental conditions for safflower production (Amjad, 2014). Pakistan expends a huge amount around US $\$ 1.5$ billion to buy in the edible oil during 2018-19. The loss of foreign exchange reserves is much less than FY 2017-18, in which about US\$ 3.0 billion were spent on import of edible oil. It is need of the time to enhance the cultivated area of the nonconventional oilseed crops like safflower and sunflower in Pakistan to meet the demand of annual vegetable oil of the country (Anonymous, 2018-19).

Present study was designed to investigate the importance of yield and its contributing parameters of a plant and to determine the high potential yielding germplasm based on different agronomic parameters by collecting and screening the national and international diverse genetic material. The selected genotypes may be included in further breeding programs enabling to help the safflower plant breeders to maintain and improve the genetic constitutions of the germplasm.

\section{Materials and Methods}

The genetic material was comprised of two hundred accessions (Table 1). Germplasm was collected from Institute of Agricultural Biotechnology and Genetic Resources (IABGR), NARC, Islamabad. Genetic material was evaluated for various yield contributing parameters during 2016-17 at experimental area of PARC Research and Training Station, Faculty of Agriculture, Bahauddin Zakariya University, Multan. Randomized Complete BlockDesign was implemented along with three replications by maintaining $15-20 \mathrm{~cm}$ interplant distance and row to row distance was 40$45 \mathrm{~cm}$. Balode et al. (2012) and Shinwari et al. (2014) screened 155 and 122 accessions of safflower for various screening purposes. All cultural practices were done as per requirement. Ten randomly chosen plants from each genotype were used to record data of the following parameters; days to flowering (DF), plant height $(\mathrm{PH})$, pods per plant (PPP), no. of primary branches per plant (PBP), no. of secondary branches per plant (SBP), thousand grain weight (TGW), yield per plant (YPP) and days to maturity (DM).

Recorded data was put to estimate the analysis of variance (Steel et al., 1997) to check the existence of significant genetic variability. Heritability $\left(\mathrm{h}^{2}\right)$ in the broad sense and genetic advance for all parameters were estimated according to the formulae as described by Allard (1960) and Falconer (1981), respectively. Principal component analysis was performed by using XLSTAT 2014.

\section{Results and Discussion}

Analysis of variance with genetic advance and heritability for 200 lines revealed valuable differences for all the traits under study (Table 2). Magnitude of genetic advance among studied parameters ranged between 11.99-27.21\% for PBP and YPP, respectively. Whereas, coefficient of variance $(\mathrm{CV})$ ranged from 2.67 to $19.71 \%$ for $\mathrm{DM}$ and $\mathrm{PBP}$, respectively. Estimation of heritability ranged between $77.4 \%$ to 99.0\% for DF and PPP in observed traits (Table 2). 
Table 1: List of safflower germplasm.

\begin{tabular}{|c|c|c|c|c|c|c|c|c|c|}
\hline Sr. No. & Accessions & Genus & Species & Origin & Sr. No. & Accessions & Genus & Species & Origin \\
\hline 1 & 016173 & Carthamus & tinctorius & India & 101 & 016327 & Carthamus & tinctorius & Afghanistan \\
\hline 2 & 016186 & Carthamus & tinctorius & India & 102 & 016329 & Carthamus & tinctorius & Afghanistan \\
\hline 3 & 016188 & Carthamus & tinctorius & India & 103 & 016331 & Carthamus & tinctorius & Afghanistan \\
\hline 4 & 016189 & Carthamus & tinctorius & India & 104 & 016333 & Carthamus & tinctorius & Afghanistan \\
\hline 5 & 016190 & Carthamus & tinctorius & India & 105 & 016334 & Carthamus & tinctorius & Afghanistan \\
\hline 6 & 016191 & Carthamus & tinctorius & India & 106 & 016335 & Carthamus & tinctorius & Afghanistan \\
\hline 7 & 016192 & Carthamus & tinctorius & India & 107 & 016337 & Carthamus & tinctorius & Afghanistan \\
\hline 8 & 016193 & Carthamus & tinctorius & India & 108 & 016338 & Carthamus & tinctorius & Afghanistan \\
\hline 9 & 016194 & Carthamus & tinctorius & India & 109 & 016341 & Carthamus & tinctorius & Iran \\
\hline 10 & 016195 & Carthamus & tinctorius & Turkey & 110 & 016342 & Carthamus & tinctorius & Iran \\
\hline 11 & 016199 & Carthamus & tinctorius & Kenya & 111 & 016343 & Carthamus & tinctorius & Iran \\
\hline 12 & 016200 & Carthamus & tinctorius & Turkey & 112 & 016345 & Carthamus & tinctorius & Iran \\
\hline 13 & 016201 & Carthamus & tinctorius & India & 113 & 016346 & Carthamus & tinctorius & Iran \\
\hline 14 & 016202 & Carthamus & tinctorius & Afghanistan & 114 & 016347 & Carthamus & tinctorius & Ethiopia \\
\hline 15 & 016203 & Carthamus & tinctorius & Afghanistan & 115 & 016349 & Carthamus & tinctorius & Portugal \\
\hline 16 & 016204 & Carthamus & tinctorius & Iran & 116 & 016351 & Carthamus & tinctorius & Portugal \\
\hline 17 & 016205 & Carthamus & tinctorius & Ethiopia & 117 & 016353 & Carthamus & tinctorius & Portugal \\
\hline 18 & 016206 & Carthamus & tinctorius & Iran & 118 & 016354 & Carthamus & tinctorius & Portugal \\
\hline 19 & 016207 & Carthamus & tinctorius & Australia & 119 & 016356 & Carthamus & tinctorius & Pakistan \\
\hline 20 & 016209 & Carthamus & tinctorius & Morocco & 120 & 016357 & Carthamus & tinctorius & Pakistan \\
\hline 21 & 016210 & Carthamus & tinctorius & Morocco & 121 & 016358 & Carthamus & tinctorius & Pakistan \\
\hline 22 & 016211 & Carthamus & tinctorius & Spain & 122 & 016359 & Carthamus & tinctorius & Pakistan \\
\hline 23 & 016216 & Carthamus & tinctorius & India & 123 & 016360 & Carthamus & tinctorius & India \\
\hline 24 & 016217 & Carthamus & tinctorius & India & 124 & 016361 & Carthamus & tinctorius & India \\
\hline 25 & 016218 & Carthamus & tinctorius & India & 125 & 016362 & Carthamus & tinctorius & India \\
\hline 26 & 016220 & Carthamus & tinctorius & Pakistan & 126 & 016364 & Carthamus & tinctorius & India \\
\hline 27 & 016225 & Carthamus & tinctorius & India & 127 & 016365 & Carthamus & tinctorius & India \\
\hline 28 & 016229 & Carthamus & tinctorius & India & 128 & 016366 & Carthamus & tinctorius & India \\
\hline 29 & 016230 & Carthamus & tinctorius & India & 129 & 016367 & Carthamus & tinctorius & India \\
\hline 30 & 016231 & Carthamus & tinctorius & India & 130 & 016368 & Carthamus & tinctorius & India \\
\hline 31 & 016233 & Carthamus & tinctorius & India & 131 & 016369 & Carthamus & tinctorius & India \\
\hline 32 & 016234 & Carthamus & tinctorius & India & 132 & 016373 & Carthamus & tinctorius & India \\
\hline 33 & 016235 & Carthamus & tinctorius & India & 133 & 016374 & Carthamus & tinctorius & Australia \\
\hline 34 & 016236 & Carthamus & tinctorius & India & 134 & 016375 & Carthamus & tinctorius & Australia \\
\hline 35 & 016237 & Carthamus & tinctorius & India & 135 & 016377 & Carthamus & tinctorius & Australia \\
\hline 36 & 016238 & Carthamus & tinctorius & India & 136 & 016379 & Carthamus & tinctorius & Australia \\
\hline 37 & 016239 & Carthamus & tinctorius & India & 137 & 016381 & Carthamus & tinctorius & Afghanistan \\
\hline 38 & 016240 & Carthamus & tinctorius & India & 138 & 016383 & Carthamus & tinctorius & Ethiopia \\
\hline 39 & 016241 & Carthamus & tinctorius & India & 139 & 016386 & Carthamus & tinctorius & Egypt \\
\hline 40 & 016242 & Carthamus & tinctorius & India & 140 & 016387 & Carthamus & tinctorius & India \\
\hline 41 & 016243 & Carthamus & tinctorius & India & 141 & 016390 & Carthamus & tinctorius & India \\
\hline 42 & 016245 & Carthamus & tinctorius & India & 142 & 016391 & Carthamus & tinctorius & India \\
\hline 43 & 016246 & Carthamus & tinctorius & India & 143 & 016392 & Carthamus & tinctorius & India \\
\hline 44 & 016247 & Carthamus & tinctorius & India & 144 & 016393 & Carthamus & tinctorius & India \\
\hline 45 & 016249 & Carthamus & tinctorius & India & 145 & 016396 & Carthamus & tinctorius & India \\
\hline 46 & 016250 & Carthamus & tinctorius & India & 146 & 016397 & Carthamus & tinctorius & India \\
\hline 47 & 016252 & Carthamus & tinctorius & India & 147 & 016398 & Carthamus & tinctorius & India \\
\hline 48 & 016253 & Carthamus & tinctorius & India & 148 & 016402 & Carthamus & tinctorius & Israel \\
\hline 49 & 016254 & Carthamus & tinctorius & India & 149 & 016407 & Carthamus & tinctorius & Iran \\
\hline 50 & 016259 & Carthamus & tinctorius & Iran & 150 & 016408 & Carthamus & tinctorius & Iran \\
\hline
\end{tabular}




\begin{tabular}{|c|c|c|c|c|c|c|c|c|c|}
\hline Sr. No. & Accessions & Genus & Species & Origin & Sr. No. & Accessions & Genus & Species & Origin \\
\hline 51 & 016260 & Carthamus & tinctorius & Egypt & 151 & 016409 & Carthamus & tinctorius & Iran \\
\hline 52 & 016261 & Carthamus & tinctorius & Egypt & 152 & 016410 & Carthamus & tinctorius & Iran \\
\hline 53 & 016262 & Carthamus & tinctorius & Egypt & 153 & 016411 & Carthamus & tinctorius & Iran \\
\hline 54 & 016264 & Carthamus & tinctorius & Egypt & 154 & 016412 & Carthamus & tinctorius & Iran \\
\hline 55 & 016265 & Carthamus & tinctorius & Pakistan & 155 & 016413 & Carthamus & tinctorius & Iran \\
\hline 56 & 016266 & Carthamus & tinctorius & Pakistan & 156 & 016414 & Carthamus & tinctorius & Iran \\
\hline 57 & 016267 & Carthamus & tinctorius & Pakistan & 157 & 016415 & Carthamus & tinctorius & Iran \\
\hline 58 & 016268 & Carthamus & tinctorius & Pakistan & 158 & 016416 & Carthamus & tinctorius & Iran \\
\hline 59 & 016269 & Carthamus & tinctorius & Pakistan & 159 & 016419 & Carthamus & tinctorius & Iran \\
\hline 60 & 016270 & Carthamus & tinctorius & Pakistan & 160 & 016420 & Carthamus & tinctorius & Iran \\
\hline 61 & 016271 & Carthamus & tinctorius & Egypt & 161 & 016421 & Carthamus & tinctorius & Iran \\
\hline 62 & 016272 & Carthamus & tinctorius & Egypt & 162 & 016423 & Carthamus & tinctorius & Iran \\
\hline 63 & 016273 & Carthamus & tinctorius & Egypt & 163 & 016425 & Carthamus & tinctorius & Iran \\
\hline 64 & 016274 & Carthamus & tinctorius & Egypt & 164 & 016426 & Carthamus & tinctorius & Turkey \\
\hline 65 & 016276 & Carthamus & tinctorius & Egypt & 165 & 016428 & Carthamus & tinctorius & Afghanistan \\
\hline 66 & 016278 & Carthamus & tinctorius & India & 166 & 016430 & Carthamus & tinctorius & Afghanistan \\
\hline 67 & 016279 & Carthamus & tinctorius & Egypt & 167 & 016431 & Carthamus & tinctorius & Afghanistan \\
\hline 68 & 016280 & Carthamus & tinctorius & Egypt & 168 & 016432 & Carthamus & tinctorius & India \\
\hline 69 & 016281 & Carthamus & tinctorius & Iran & 169 & 016434 & Carthamus & tinctorius & India \\
\hline 70 & 016283 & Carthamus & tinctorius & Iran & 170 & 016435 & Carthamus & tinctorius & India \\
\hline 71 & 016284 & Carthamus & tinctorius & Iran & 171 & 016436 & Carthamus & tinctorius & India \\
\hline 72 & 016285 & Carthamus & tinctorius & Iran & 172 & 016438 & Carthamus & tinctorius & India \\
\hline 73 & 016287 & Carthamus & tinctorius & Iran & 173 & 016439 & Carthamus & tinctorius & India \\
\hline 74 & 016288 & Carthamus & tinctorius & Iran & 174 & 016441 & Carthamus & tinctorius & Sudan \\
\hline 75 & 016289 & Carthamus & tinctorius & Iran & 175 & 016442 & Carthamus & tinctorius & Sudan \\
\hline 76 & 016290 & Carthamus & tinctorius & Iran & 176 & 016443 & Carthamus & tinctorius & Sudan \\
\hline 77 & 016291 & Carthamus & tinctorius & Iran & 177 & 016446 & Carthamus & tinctorius & Russia \\
\hline 78 & 016292 & Carthamus & tinctorius & Iran & 178 & 016447 & Carthamus & tinctorius & Egypt \\
\hline 79 & 016293 & Carthamus & tinctorius & Iran & 179 & 016451 & Carthamus & tinctorius & Egypt \\
\hline 80 & 016295 & Carthamus & tinctorius & Iran & 180 & 016453 & Carthamus & tinctorius & Egypt \\
\hline 81 & 016296 & Carthamus & tinctorius & Iran & 181 & 016458 & Carthamus & tinctorius & India \\
\hline 82 & 016297 & Carthamus & tinctorius & Iran & 182 & 016459 & Carthamus & tinctorius & India \\
\hline 83 & 016298 & Carthamus & tinctorius & Iran & 183 & 016460 & Carthamus & tinctorius & India \\
\hline 84 & 016299 & Carthamus & tinctorius & Iran & 184 & 016464 & Carthamus & tinctorius & India \\
\hline 85 & 016301 & Carthamus & tinctorius & Iran & 185 & 016465 & Carthamus & tinctorius & India \\
\hline 86 & 016303 & Carthamus & tinctorius & Iran & 186 & 016466 & Carthamus & tinctorius & India \\
\hline 87 & 016304 & Carthamus & tinctorius & Iran & 187 & 016467 & Carthamus & tinctorius & India \\
\hline 88 & 016306 & Carthamus & tinctorius & Iran & 188 & 016469 & Carthamus & tinctorius & India \\
\hline 89 & 016308 & Carthamus & tinctorius & Iran & 189 & 016470 & Carthamus & tinctorius & Turkey \\
\hline 90 & 016310 & Carthamus & tinctorius & Iran & 190 & 016471 & Carthamus & tinctorius & Iran \\
\hline 91 & 016312 & Carthamus & tinctorius & Turkey & 191 & 016474 & Carthamus & tinctorius & Iran \\
\hline 92 & 016313 & Carthamus & tinctorius & Turkey & 192 & 016478 & Carthamus & tinctorius & Iran \\
\hline 93 & 016314 & Carthamus & tinctorius & Turkey & 193 & 016479 & Carthamus & tinctorius & Iran \\
\hline 94 & 016316 & Carthamus & tinctorius & Turkey & 194 & 016482 & Carthamus & tinctorius & Iran \\
\hline 95 & 016317 & Carthamus & tinctorius & Turkey & 195 & 016483 & Carthamus & tinctorius & USA \\
\hline 96 & 016318 & Carthamus & tinctorius & Spain & 196 & 016484 & Carthamus & tinctorius & USA \\
\hline 97 & 016320 & Carthamus & tinctorius & Germany & 197 & 016489 & Carthamus & tinctorius & China \\
\hline 98 & 016324 & Carthamus & tinctorius & Iraq & 198 & 016492 & Carthamus & tinctorius & China \\
\hline 99 & 016325 & Carthamus & tinctorius & Iraq & 199 & 016495 & Carthamus & tinctorius & China \\
\hline 100 & 016326 & Carthamus & tinctorius & Iraq & 200 & 016501 & Carthamus & tinctorius & USA \\
\hline
\end{tabular}


Table 2: Means and analysis of variance (ANOVA) for eight traits among 200 safflower genotypes.

$\begin{array}{llllllll}\text { Parameters } & \text { MS (R) } & \text { MS (V) } & \text { MS(E) } & \text { Means } \pm \text { SE } & \mathbf{h}^{\mathbf{2}(\%)} & \text { GA (\%) } & \text { CV(\%) } \\ \text { DF } & 9.512 & 50.028 & 11.294 & 121.97 & 77.4 & 22.22 & 2.755 \\ \text { PH } & 8.202 & 567.962 & 13.287 & 104.77 & 97.7 & 19.6 & 3.479 \\ \text { DM } & 9.052 & 87.593 & 16.453 & 151.70 & 81.2 & 17.33 & 2.673 \\ \text { PPP } & 1.872 & 224.181 & 2.223 & 38.31 & 99.0 & 16.51 & 3.892 \\ \text { PBP } & 0.140 & 4.902 & 0.246 & 2.57 & 95.0 & 11.99 & 19.31 \\ \text { SBP } & 1.415 & 74.672 & 1.1369 & 8.54 & 98.5 & 23.51 & 12.48 \\ \text { TGW } & 5.612 & 157.537 & 4.012 & 37.78 & 97.5 & 14.73 & 5.301 \\ \text { YPP } & 15.247 & 1248.901 & 20.240 & 83.82 & 98.4 & 27.21 & 5.367\end{array}$

DF: days to flowering; PH: plant height; DM: days to maturity; PPP: pods per plant; PBP: number of primary branches per plant; SBP: number of secondary branches per plant; TGW: thousand grain weight; YPP: yield per plant; MS(R): mean square of replications; $M S(V)$ : mean square of varieties; $M S(E)$ : mean square of errors; $b^{2}$ : heritability; GA: genetic advance; $G V$ : coefficient of variability.

Table 3: Genotypic ( $r$ ) and phenotypic ( $r$ ) correlation between various morpho-physiological traits of saflower.

\begin{tabular}{|c|c|c|c|c|c|c|c|c|}
\hline Traits & DF & PH & DM & PPP & PBP & SBP & TGW & YPP \\
\hline \multicolumn{9}{|l|}{ DF } \\
\hline G & 1.000 & $-0.0279^{*}$ & $0.9275^{*}$ & $0.0325^{*}$ & $-0.0992^{*}$ & $-0.0371^{*}$ & $0.0679^{*}$ & $-0.0168^{*}$ \\
\hline $\mathrm{P}$ & 1.000 & -0.0246 & $0.7489^{* *}$ & 0.0271 & -0.0770 & -0.0300 & 0.0561 & -0.0619 \\
\hline \multicolumn{9}{|l|}{$\mathrm{PH}$} \\
\hline G & & 1.0000 & $-0.1590^{*}$ & $0.6102^{*}$ & $-0.783^{*}$ & $-0.0690^{*}$ & $0.1032^{*}$ & $0.6551^{*}$ \\
\hline $\mathrm{P}$ & & 1.0000 & -0.1399 & $0.5999^{*}$ & $-0.0784^{*}$ & $-0.0677^{*}$ & 0.0993 & $0.6423^{* *}$ \\
\hline \multicolumn{9}{|l|}{ DM } \\
\hline G & & & 1.0000 & $-0.0989^{*}$ & -0.0055 & -0.0085 & $-0.0670^{*}$ & $-0.1165^{*}$ \\
\hline$P$ & & & 1.0000 & -0.0847 & -0.0019 & -0.0047 & -0.0582 & -0.1051 \\
\hline \multicolumn{9}{|l|}{ PPP } \\
\hline G & & & & 1.0000 & -0.0005 & $-0.0694^{*}$ & $0.1099^{*}$ & $0.7520^{*}$ \\
\hline $\mathrm{P}$ & & & & 1.0000 & 0.0014 & -0.0670 & 0.1082 & $0.7437^{* *}$ \\
\hline \multicolumn{9}{|l|}{ PBP } \\
\hline G & & & & & 1.0000 & $0.7807^{*}$ & $-0.1782^{*}$ & $-0.0826^{*}$ \\
\hline $\mathrm{P}$ & & & & & 1.0000 & $0.7541^{* *}$ & -0.1666 & -0.0791 \\
\hline \multicolumn{9}{|l|}{ SBP } \\
\hline G & & & & & & 1.0000 & $-0.1445^{*}$ & -0.1388 \\
\hline $\mathrm{P}$ & & & & & & 1.0000 & -0.1432 & -0.1367 \\
\hline \multicolumn{9}{|l|}{ TGW } \\
\hline G & & & & & & & 1.0000 & $0.1231^{*}$ \\
\hline $\mathrm{P}$ & & & & & & & 1.0000 & 0.1222 \\
\hline \multicolumn{9}{|l|}{ YPP } \\
\hline G & & & & & & & & 1.0000 \\
\hline$P$ & & & & & & & & 1.0000 \\
\hline
\end{tabular}

For abbreviations, See Table 2; "Significant; ** Highly significant.

Genotypic and phenotypic correlations were observed among eight (8) morpho-physiological parameters in safflower. Days to flowering (DF) showed negatively significant interrelationship with $\mathrm{PH}, \mathrm{PBP}, \mathrm{SBP}$ and YPP, while positive significant interrelationship was observed among DF, DM, PPP and TGW. Plant height $(\mathrm{PH})$ showed negatively significant genotypic and phenotypic interrelations with $\mathrm{DM}, \mathrm{PBP}$ and SBP. However, there was positive and significant genotypic interrelation among PH, PPP, TGW and YPP. Negatively significant genotypic and phenotypic interrelations were present among days to maturity and other yield related parameters. Pods per plant (PPP) had negatively significant interrelation with 
PBP and SBP, while positively significant correlation was observed among PPP, TGW and YPP. Results revealed the existence of positive correlation among PBP and SBP, while both traits showed negative interrelation with TGW and YPP. However, TGW showed positive interrelation with YPP.

\section{Principal component analysis}

Results of PCA showed that on basis of eigen value, data is considered up to three principal components. It was noted that $74 \%$ variability of the total variation lies in three PC's. First PC has $30.6 \%$ variability, while PC2 and PC3 has $22.9 \%$ and $20.5 \%$ variability of the total existing variation of the data. These PC's are orthogonal with each other. In first PC, four parameters i.e. PH, PPP, TGW and YPP were correlated with each other in negative direction, while remaining traits showed positive correlation with each other. In PC2, five yield related parameters viz; PH, PPP, PBP, SBP and YPP were negatively correlated with each other, while remaining traits were positively interrelated with each other. Under PC3, only one parameter (TGW) showed negative correlation with other parameters, which is a valuable yield index. The component with eigenvalues > 1 contributed $74 \%$ (Table 4) of the total variability among accessions of safflower for various morph-physiological traits. Two hundred accessions of safflower have been divided into eight cluster groups on the basis of their performance for studied parameters. Cluster 7 contain maximal (41) number of accessions, while cluster number 5 and 6 consist of lowest (15) number of accessions each. Cluster number 1 and 3 consist of 34 and 28 accessions respectively. While cluster number 4, 2 and 8 comprised of 26, 21 and 20 accessions respectively (Table 5).

Results of ANOVA revealed the presence of high genetic variability among all accessions of safflower for studied parameters and proved that data was fit for further statistical analysis. Existence of variation is useful for various genetic analysis and ultimately helpful in selection and improvement of crop (Kose et al., 2018). The basic purpose of correlation studies was to observe a common relationship between different characters and their level of the involvement to the yield (Panhwar et al., 2003). Plant parameters viz., DF, DM, PBP and SBP showed negative impact on yield and grain yield reduces with increase in number of DF, DM, PBP and SBP. However, plant attributes like PH, PPP and TGW had positive effect on yield of the crop as yield increases with increase in magnitude of these traits. It was observed that grain yield had significant interaction with $\mathrm{PPP}, \mathrm{PH}$ and TGW (Ahmadzadeh, 2013; Kose et al., 2018). To intensify the crop yield, magnitude of plant attributes like PPP and TGW must be increased because these parameters had direct influence on YPP (Elfadl et al., 2010; Eslam et al., 2010; Safavi, 2011). Based upon the results of correlation, it is suggested that genotypes having higher magnitude of $\mathrm{PH}$, branches per plant and grain weight will be selected for future breeding program to enhance yield (Kose et al., 2018). If value of ' $r$ ' (correlation) is near to 1 , interrelation among two variables is positive and traits are highly dependent on each other. If ' $r$ ' is nearly zero among different variables no interdependency is observed, while ' $r$ ' with negative sign among variables proved negative relation among variables (Katar, 2013).

$\begin{array}{llll}\text { Table 4: Principal Component } & \text { Analysis } & \text { (PCA) } \\ \text { germplasm. } & & & \\ & \text { PC 1 } & \text { PC 2 } & \text { PC 3 } \\ \text { Eigen values } & 2.448 & 1.832 & 1.640 \\ \text { Proportion of variance } & 30.600 & 22.903 & 20.497 \\ \text { Cumulative variance } & 30.600 & 53.503 & 74.000 \\ \text { Eigen vectors } & & & \\ \text { Variables } & \text { PC 1 } & \text { PC 2 } & \text { PC 3 } \\ \text { DF } & 0.108012 & 0.674046 & 0.644409 \\ \text { PH } & -0.808049 & -0.111115 & 0.199538 \\ \text { DM } & 0.255235 & 0.634851 & 0.640596 \\ \text { PPP } & -0.831564 & -0.104359 & 0.308155 \\ \text { PBP } & 0.287557 & -0.685164 & 0.554232 \\ \text { SBP } & 0.331518 & -0.656911 & 0.549469 \\ \text { TGW } & -0.263355 & 0.214418 & -0.143479 \\ \text { YPP } & -0.874539 & -0.068448 & 0.222903\end{array}$

Estimation of heritability is a promising indication about the transmittance of various parameters from parents to progeny. Appraisal of heritability is very helpful in selection of suitable genotypes/ accessions among various environmental and field conditions from a heterogeneous breeding population (Tahernezhad et al., 2018). Based upon percent (\%) magnitude, heritability could be classified into low (0-30\%), medium (30-60\%) and high (>60\%) (Reddy et al., 2013). Results revealed that magnitude of heritability was greater than $70 \%$ for all studied parameters (Table 2), indicating high transmittance percentage. It is also predicted that these parameters 
Table 5: Cluster-wise accession membership.

\section{Cluster No. Accessions}

I

A5, A9, A23, A24, A29, A35, A50, A52, A53, A54, A55, A78, A79, A86, A90, A96, A99, A100, A102, A105, A108, A109, A114, A118, A121, A122, A131, A137, A141, A150, A162, A163, A185, A189

II

A33, A41, A48, A58, A59, A73, A85, A97, A101, A106, A110, A119, A142, A143, A144, A164, A172, A175, A177, A182, A190

III

A14, A15, A16, A17, A28, A32, A37, A43, A47, A56, A57, A69, A70, A74, A84, A91, A92, A94, A98, A104, A111, A112, A115, A117, A126, A128, A132, A138

IV

A2, A3, A10, A13, A27, A36, A38, A40, A60, A63, A64, A65, A83, A87, A93, A95, A103, A107, A113, A124, A129, A133, A134, A135, A136, A139

V A7, A8, A11, A18, A20, A21, A26, A34, A42, A49, A62, A68, A71, A72, A82

VI A1, A6, A12, A19, A22, A25, A30, A31, A39, A61, A66, A67, A123, A127, A140

VII A4, A44, A45, A46, A51, A75, A76, A77, A80, A81, A88, A89, A116, A120, A125, A130, A145, A148, A149, A152, A153, A154, A155, A156, A158, A159, A160, A161, A165, A166, A169, A173, A174, A178, A179, A186, A191, A193, A196, A199, A200

VIII A146, A147, A151, A157, A167, A168, A170, A171, A176, A180, A181, A183, A184, A187, A188, A192, A194, A195, A197, A198

are less influenced by environment and highly suitable for early selection due to presence of additive nature of genetic inheritance. These results are in accordance with the findings of Arslan (2007), Sirisha (2009) and Elfadl et al. (2010).

PCA is a multivariate analysis technique, which is usually used to develop coordinated axis of an orthogonal and to estimate the relative importance of classified variables. This technique is characterized by conversion of complex plant data analysis into simple form (Slavkovic et al., 2004). Maximum variation was observed among first three PC's and it contained 74\% of the total variability. Ahmadzadeh (2013) reported that 72.92 percent of the total variation was found in first three PC's, while Kose et al. (2018) found 65.4 percent of total variation in first two PC's. If the eigen values are greater than one, then diversity is not found in the traits and values are less than one, then diversity is found in all the characters. Negative eigen values were ignored because these values have no importance while positive values considered diversity is found in the characters. Cluster analysis classified the germplasm into eight groups on basis of similarity in their mean performance for observed parameters. Cluster analysis is a helpful technique to categorize the germplasm into well-defined subgroups and groups depending upon resemblance and deviation among mean performance of observed parameters (Biljana and Onjia, 2007).

\section{Conclusions and Recommendations}

Significant genetic variability was observed among germplasm regarding yield and its related attributes. The highest estimates of genetic advance and heritability for all traits showed their significance in selection of particular parents to be used in future breeding program. Higher heritability magnitude showed the predominance of additive genetic effects for studies traits, due to which direct and early selection is useful. From the results of correlation, it was concluded that three parameters viz., plant height, pods per plant and thousand grain weight had positively significant genotypic interrelationship with yield per plant. So, genotypes having higher value of PH, PPP and TGW can be selected to develop high yielding safflower varieties for Pakistan.

\section{Acknowledgment}

This study supported by the PARC, Government of Pakistan. We pay our gratitude to Dr. Yusuf Zafar (T.I), worthy chairman PARC and IABGR (Institute), NARC, Islamabad for supplying seeds of safflower accessions. We are thankful to Dr. Sohail (Department of Statistics), who provided computer programs for statistical analysis.

\section{Novelty Statement}

Safflower is a good source of high-quality oil and mostly used for food, industry, ornamental purpose and as a biofuel. Pakistan expands a huge amount of foreign exchange on import of oil. By evaluation of available germplasm in the country, we will be able to develop high yielding safflower varieties with less 
input resources. This will not only helpful to meet the oil requirement of the country but also to improve health of the people and save the foreign exchange.

\section{Author's Contributions}

Rao Wali Muhammad and Abdul Qayyum planed the proposal and conducted the whole study. Methodology and analysis were carried out by Muhammad Qadir Ahmad and Waqas Malik. Hafiz Muhammad Wasif Ali completed the manuscript write up. Overall assessment of write up was done by Amir Hamza. Statistical analysis was accomplished by Etrat Noor.

\section{Conflict of interest}

The authors have declared no conflict of interest.

\section{References}

Ahmadzadeh, A., 2013. Genetic diversity and classification of spring safflower (Carthamus tinctorius L) cultivars using morphological characters. Adv. Biores., 4(4): 125-131.

Allard, R.W., 1960. Principles of Plant Breeding. John Wiley and Sons Inc., New York, USA. pp. 485.

Amjad, M., 2014. Oilseed crops of Pakistan: Status paper. Pak. Agric. Res. Council Islamabad, (PARC). pp. 1-40. https://doi.org/10.18356/ a55dee27-en

Anonymous, 2018-19. Pakistan bureau of statistics, ministry of finance. Govt. of Pakistan, Islamabad.

Arslan, B., 2007. Assessing of heritability and variance components of yield and some agronomic traits of different safflower (Carthamus tinctorius L.) cultivars. Asian Plant Sci., 6(3): 554- 557. https://doi.org/10.3923/ ajps.2007.554.557

Arslan, B., F. Altuner and M. Tuncturk. 2003. An investigation on yield and yield components of some safflower varieties which grown in Van. $5^{\text {th }}$ Field Crops Cong. Turkey, 1: 468-472.

Balode, K.L., P.N. Mane, P.K. Rathod and S.N. Deshmukh. 2012. Evaluation of safflower germplasm for resistant to Alternaria leaf spot. J. Oilseeds Res., 29: 97-99.

Baydar,H. and I. Turgut.1999. Some morphological composition of fatty acids in oilseed plants and change according to physiological properties and ecological regions. Turk. J. Agric. For., 23(1): 81-86.

Biljana, S. and A. Onjia.2007. Multivariate analyses of microelement contents in wheat cultivated in Serbia. Food Contr., 18(4): 338-345. https:// doi.org/10.1016/j.foodcont.2005.10.017

Canavar, O., K.P. Gotz, Y.O. Koca and F. Ellmer. 2014. Relationship between water use efficiency and $\delta 13 \mathrm{c}$ isotope discrimination of safflower (Carthamus tinctorius L.) under drought stress. Turk. J. Field Crops. 19(2): 212-220. https:// doi.org/10.17557/tjfc.28375

Conge, B., B. Gürbüz and M. ve Kıralan. 2007. Oil content and fatty acid composition of some safflower (Carthamus tinctorius L.) varieties sown in spring and winter. Int. J. Natur. Eng. Sci., 1(3): 11-15.

Elfadl, E., C. Reinbreeht and W. Claupein. 2010. Evaluation of phenotypic variation in a worldwide germplasm collection of safflower (Carthamus tinctorius L.) grown under organic farming conditions in Germany. Genet. Resour. Crop Evol., 57(2): 155-170. https://doi. org/10.1007/s10722-009-9458-7

Emongor, V.E., 2010. Safflower (Carthamus tinctorius L.) the underutilized and neglected crop: A review.Asian J.Plant Sci., 9(6): 299-306. https://doi.org/10.3923/ajps.2010.299.306

Emongor, V.E. and O. Oagile. 2017. Safflower production. The Botswana University of Agriculture and Natural Resources, Gaborone Botswana. pp. 1-67.

Emongor, V.E., O. Oagile and B. Kedikanetswe. 2015. Effects of plant population and season on growth and development of safflower (Carthamus tinctorius L.) as an ornamental plant. Acta Hortic., 1077: 35-45. https://doi. org/10.17660/ActaHortic.2015.1077.3

Eslam, B.P., H. Monirifar and M.T. Ghassemi. 2010. Evaluation of late season drought effects on seed and oil yields in spring safflower genotypes. Turk. J. Agric., 34(5): 373-380.

Falconer, D.S., 1981. Introduction to quantitative genetics. $2^{\text {nd }}$ Edition, Longman Group Ltd., London. pp. 1-133.

Hojati, M., S.A.M. Modarress-Sanavy, M. Karimi and F. Ghanati. 2011. Responses of growth and antioxidant systems in Carthamus tinctorius L. under water deficit stress. Acta Physiol. Plant, 33(1): 105-112. https://doi.org/10.1007/ s11738-010-0521-y 
Istanbulluoglu, A., 2009. Effects of irrigation regimes on yield and water productivity of safflower (Carthamus tinctorius L.) under Mediterranean climatic condition. Agric. Water Manage., 96(12): 1792-1798. https:// doi.org/10.1016/j.agwat.2009.07.017

Katar,D.,2013. Determination of efficiency of yield components on oil yield per plant in safflower breeding by different statistical methods. Glob. J. Sci. Front. Res. Agric. Vet. Sci., 13(8): 11-20.

Kose, A., A. Onder, O. Bilir and F. Kosar. 2018. Application of multivariate statistical analysis for breeding strategies of spring safflower (Carthamus tinctorius L.). Turk. J. Field Crops. 23(1): 12-19. https://doi.org/10.17557/ tjfc. 413818

Panhwar, R.N., H.K. Keerio, Y.M. Memon, S. Junejo, M.Y. Arain, M. Chohan, A.R. Keerio and B.A. Abro. 2003. Response of Thatta-10 sugarcane variety to soil and foliar application of zinc sulphate $\left(\mathrm{ZnSO} 4.7 \mathrm{H}_{2} \mathrm{O}\right)$ under half and full doses of NPK fertilizer. J. Appl. Sci., 3(4): 266-269. https://doi.org/10.3923/ jas.2003.266.269

Reddy, M.P., B.N. Reddy, B.T. Arsul and J.J. Maheshwari. 2013. Genetic variability, heritability and genetic advance of growth and yield components of linseed (Linum usitatissimum L.). Int. J. Curr. Microbiol. App. Sci., 2(9): 231-237.

Reza, A.M., M.J. Mirhadi, B. Delkhosh and A. Omidi. 2013. Evaluation of native and exotic safflower (Carthamus tinctorius L.) genotypes for some important agronomic traits and fatty acid composition. Ann. Biol. Res., 4(6): 200204.

Safavi, S.M., 2011. Heritability and genetic gain of some morphological traits in safflower (Carthamus tinctorius L.). Am. J. Sci. Res., 13: 14-18.
Sehgal, D., S.N. Raina, R.M. Devarumatha, T. Sasanuma and T. Sasakuma. 2009. Nuclear DNA assay in solving issues related to ancestry of the domesticated diploid safflower (Carthamus tinctorius L.) and the polyploid (Carthamus) taxa, and phylogenetic and genomic relationships in the genus Carthamus L. (Asteraceae). Mol. Phylogenet., 53(3): 631-644. https://doi. org/10.1016/j.ympev.2009.07.012

Shinwari, Z.K., H. Rehman and M.A. Rabbani. 2014. Morphological traits based genetic diversity in safflower (Cathamus tinctorius L.). Pak. J. Bot., 46(4): 1389-1395.

Sirisha, M.,2009. Studies on genetic divergence and character association in safflower (Carthamus tinctorius L.). M.Sc. thesis, Acharya N.G. Ranga Agric. Univ. India.

Slavkovic, L., B. Skrbic, N. Miljevic and A. Onjia. 2004. Principal component analysis of trace elements in industrial soils. Environ. Chem. Lett., 2(2):105-108. https://doi.org/10.1007/ s10311-004-0073-8

Steel, R.G.D., J.H. Torrie and D.A. Dickey. 1997. Principles and procedures of statistics: A biometrical approach. $3^{\text {rd }}$ ed. McGraw Hill Book Co. Inc. New York. pp. 400-428.

Tahernezhad, Z., J. Saba, M. Zein-al-abedini, S.S. Pourdad, and M.R. Ghaffari. 2018. Estimation of broad-sense heritability and variance components for seed yield and agronomic traits in native and exotic safflower (Carthamus tinctorius L.) genotypes. Bangladesh J. Bot., 47(3): 501-508. https://doi.org/10.3329/bjb. v47i3.38718

Velasco, L., B. Perez-Vich and J.M. FernandezMartinez. 2005. Identification and genetic characterization of a safflower mutant with modified tocopherol profile. Plant Breed, 124(5): 459-473. https://doi.org/10.1111/ j.1439-0523.2005.01150.x 